\title{
Ultra-miniature bluetooth tag with antenna on package for red palm weevil tracking
}

\author{
Rana Muhammad Bilal, Zulma Lopez Reyes, and Atif Shamim \\ Department of Electrical and Computer Engineering, CEMSE Division \\ King Abdullah University of Science and Technology (KAUST) \\ Thuwal, Saudi Arabia \\ ranamuhammad.bilal@kaust.edu.sa
}

\begin{abstract}
Ultra-small tracking devices are necessary for monitoring small insects or pests such as Red Palm Weevil (RPW). Understanding movement and behavior of such pests is important because they create huge economic impact by destroying expensive crops. However, designing RF system and antenna mountable on such small insects is very challenging. Previously reported works in literature either have insufficient tracking range of few $\mathrm{cm}$ only; or are bulky (greater than $4 \mathrm{~cm}$ ); or are only capable of tracking one insect at a time. This paper reports a Bluetooth enabled ultra-miniature tracking tag which is detectable at a range of $78 \mathrm{~m}$ and can be used to track multiple insects simultaneously. Presented tag uses a modified patch antenna-on-package design, and measures $6 \mathrm{~mm} \times 6 \mathrm{~mm} \times 3.5 \mathrm{~mm}$ only.
\end{abstract}

Keywords-insect tracking, RPW tracking, BLE tracking tag, antenna on package, modified patch antenna.

\section{INTRODUCTION}

Red Palm Weevil (RPW) is a destructive pest for palm trees. Entire lifespan of its larvae is spent in palm tree's trunk, hollowing it out and resulting in tree's death. Over past few decades, Red Palm Weevil has spread to major part of Middle East, where date palms form a significant portion of exports and revenue. This has fueled interest in understanding RPW behavior to formulate counter strategies and control policies. For instance, understanding average and maximum commute distance of RPW adults helps to establish quarantine radius i.e. how much potentially infected area to cordon off if one date palm is found infected in a date farm. However, designing an RF system and antenna within such small size and weight limitations (so as to be suitable for attaching to RPW) is challenging.

RPW adults typically have body length of $35 \mathrm{~mm}$ to $40 \mathrm{~mm}$. For tracking such insects, most suitable place to attach a tag is "thorax", as indicated in Fig. 1 below. Attaching tag to thorax does not blocks any moving part such as wings or leg; or critical sensory organ such as eyes or antennae; and thus does not hinders natural movement/behavior of the insect. On thorax of an adult red palm weevil an approximate $8 \mathrm{~mm}$ by $8 \mathrm{~mm}$ is available, where a tracking tag might be attached. This area is marked in yellow in Fig. 1.

As discussed in [1], device attachment to insects should weigh less than 10 percent of body weight of the insect. Typical RPW adult weight is $1 \mathrm{~g}$ approximately [2]. Thus, a tracking tag suitable for monitoring RPWs should be maximum $8 \mathrm{~mm} \times 8 \mathrm{~mm}$ in size and not weigh more than $100 \mathrm{mg}$. Further, in order to provide meaningful monitoring information, tag must have adequate detection range (at least few tens of meter).

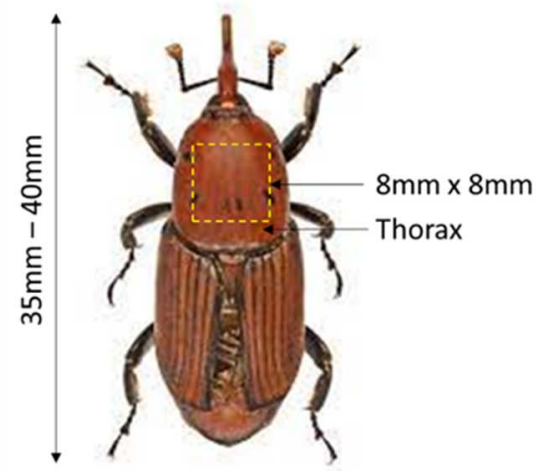

Fig. 1. Typical adult red palm weevil, area suitable for tracking tag mounting indicated by yellow box

Certain previous works in research literature have reported systems or approaches for tracking of such small insects. For instance [3] is a passive Radio Frequency Identification (RFID) tag for tracking/monitoring of honey bees. This tag although small in size and weight, has a very small tracking range of few cm only and thus is only suitable for very specific application of honey bee tracking, where an RFID reader can be fitted on nest's entrance. Reference [4] has reported millimeter wave harmonic sensor and radar system for tracking of Australian plague locusts. However, harmonic sensors do not contain identification information and thus cannot be used to track multiple insects simultaneously. Reference [5] has reported a very high frequency (VHF) active RFID tag for insects tracking. However, the reported tag requires a $10 \mathrm{~cm}$ long thin wire whip antenna, which makes it unsuitable for mounting on small insects such as RPWs. Finally, [6] has used Bluetooth Low Energy protocol combined with Antenna on package design to present a $6 \mathrm{~mm}$ x $6 \mathrm{~mm} \times 5 \mathrm{~mm}$ tracking tag for monitoring of RPWs, with identification information as well as a read range of $300 \mathrm{~m}$. Whilst the tag presented in [6] is decently small, it misses on certain Bluetooth low energy protocol aspects that can be exploited to relax bandwidth requirements on Antenna design and thereby further reduce size of the Tag. Also, reported range of $300 \mathrm{~m}$ is in free space and doesn't takes into account ground reflection or multipath effect.

This paper presents design of a Bluetooth enabled ultraminiaturized tracking tag for RPW insects, with a modified patch antenna on package. Presented tag exploits Bluetooth low energy protocol to relax bandwidth requirement of antenna design from $83.5 \mathrm{MHz}$ to $2 \mathrm{MHz}$, measures $6 \mathrm{~mm} \mathrm{x}$ $6 \mathrm{~mm} \times 3.5 \mathrm{~mm}$ and has a detection range of $78 \mathrm{~m}$ (including consideration for multipath). Body effect of mounting on RPW is also discussed. 


\section{TAG DESIGN}

Bluetooth based tracking tag presented in this paper, consists of an embedded BLE printed circuit board (PCB), a modified patch antenna on package and a silver oxide coin cell to power the tag. Tag is programmed with an identification number, which is meant to be unique for each tag. Each tag broadcasts its unique identification number at a programmed broadcast interval of $100 \mathrm{~ms}$. Thus, a BLE scanning device can scan for these advertisement packets and identify vicinity of multiple RPW insects simultaneously, each distinguished by its unique identification number.

\section{A. Bluetooth low energy (BLE) $P C B$}

Embedded PCB for the tracking tag uses NRF52832, which is a Bluetooth system-on-chip (SOC) in a wafer level chip scale package (WLSCP). Footprint for this SOC measures $3 \mathrm{~mm} \times 3.2 \mathrm{~mm}$ only. A 2 layer printed circuit board containing requisite passives i.e. a $32 \mathrm{MHz}$ crystal oscillator to generate clock pulse for the circuit, decoupling capacitors and programming pads is designed as shown in Fig. 2 below.

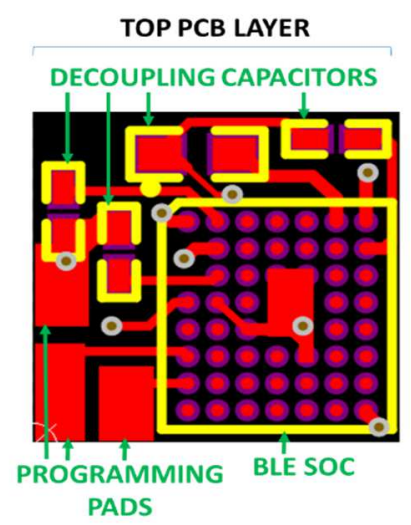

BOTOM PCB LAYER

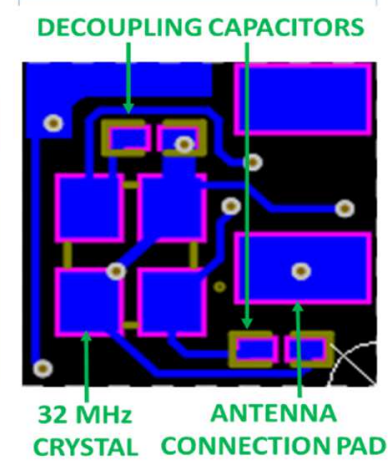

Fig. 2. Top and Bottom copper layers of Embedded PCB

Fabricated PCB measures $5 \mathrm{~mm} \times 5 \mathrm{~mm}$ and weighs $57.7 \mathrm{mg}$ only. Fig. 3 shows fabricated PCB of tracking tag, its size comparison to a human finger nail and an RPW insect fitted with the fabricated PCB using super glue.

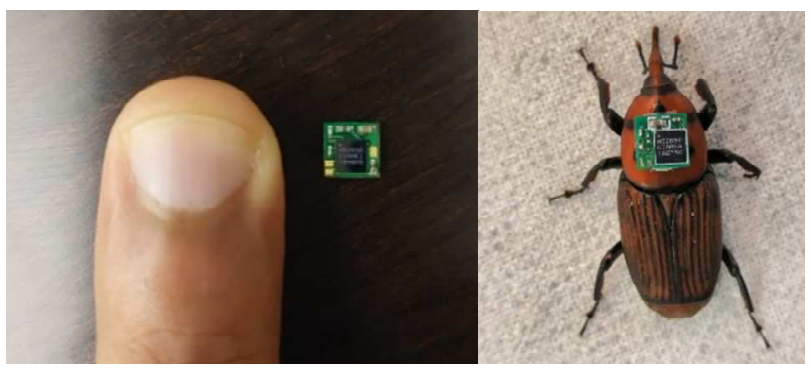

Fig. 3. Fabricated PCB of tracking device, size comparison to a human finger nail, an RPW mounted with fabricated PCB

\section{B. BLE protocol exploit for antenna bandwidth reduction}

BLE protocol specifies spectrum from $2400 \mathrm{MHz}$ to 2483.5MHz. Covering this entire bandwidth can make antenna design (in a constrained space of less than $8 \mathrm{~mm} x$ $8 \mathrm{~mm}$ ) quite challenging. Interestingly however, BLE spectrum is sub-divided into 37 data and 3 advertisement channels. As per BLE specifications, a peripheral device may advertise in non-connectable mode. Thus, such a device only utilizes the $2 \mathrm{MHz}$ advertisement channels and is discoverable/detectable on nearby BLE scanning devices. Tag presented in this paper is configured via embedded programming to advertise in non-connectable peripheral mode at $39^{\text {th }}$ BLE channel. Thus, the associated bandwidth requirement for antenna design relaxes to $2 \mathrm{MHz}$ (from $2479 \mathrm{MHz}$ to $2781 \mathrm{MHz}$ )

\section{Modified Patch Antenna-on-Package}

Two techniques are implemented to achieve the high level of miniaturization that this application demands: dielectric loading and shaping. First, the antenna is designed on a high dielectric constant material, so the wave propagation slows down [7]. Subsequently, four slots are introduced to force the current to travel a longer path, and vertical sections of conductor are added to extend the size of the patch [8]. Fig. 4 displays the development of the geometry and table 1 summarizes the performance at each stage.

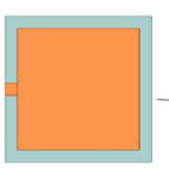

1

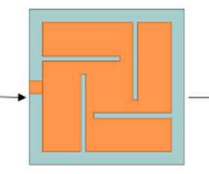

2

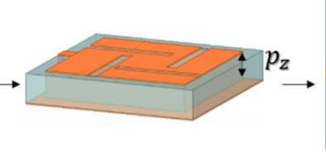

3

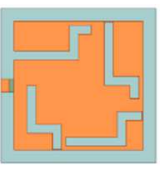

4
Fig. 4. Antenna design stages.

The antenna is a $5 \times 5 \mathrm{~mm}^{2}$ patch antenna on a $1 \mathrm{~mm}$ thick ceramic dielectric with relative permittivity of 37 , and loss tangent of 0.001 , and a $6 \times 6 \mathrm{~mm}^{2}$ ground plane at the bottom. The patch is fed on the edge to ensure high impedance. At the first stage, the antenna operates at $4.8 \mathrm{GHz}$, so the patch is to be modified to lower the frequency.

In the second stage, four straight slots are introduced, and the effect of their width $S_{w}$ and length $S_{1}$ are studied. As shown in Fig. 5, the frequency shifts down as the width or length increases because the current path is lengthen. However, the antenna does not reach the desired frequency range yet.

In the third stage, three vertical sections are added to enlarge the radiating structure. Similarly to the previous case, the frequency shifts down as the height of the vertical section $\mathrm{p}_{\mathrm{z}}$ increases, reaching $2.6 \mathrm{GHz}$ when $\mathrm{p}_{\mathrm{z}}$ becomes $0.9 \mathrm{~mm}$.

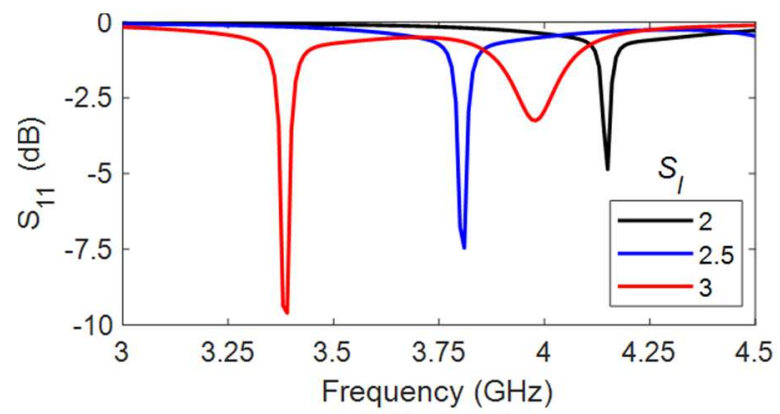

a) Slot length

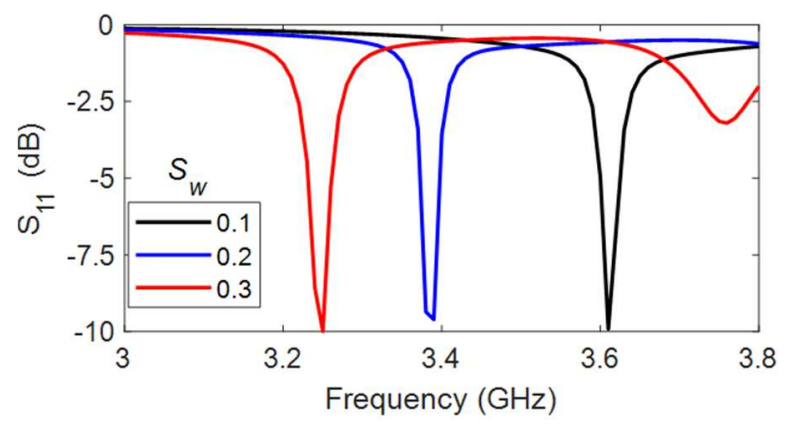

b) Slot Width 


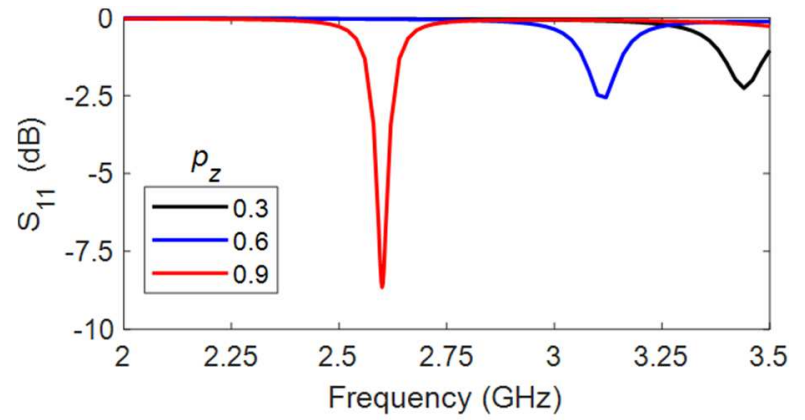

c) Height of the vertical section

Fig. 5. Return loss of the parametric simulations. (Dimensions are in $\mathrm{mm}$ )

In order to achieve further miniaturization the slots are bended and lengthen, and a $2.7 \mathrm{nH}$ inductor is inserted to match the antenna to $50 \Omega$. The practical losses of the inductor are considered in the simulation as a series resistor of $0.13 \Omega$. $\mathrm{Up}$ to this stage, the antenna resonates at $2445 \mathrm{MHz}$ and its maximum gain is $-14.8 \mathrm{~dB}$.

TABLE I. ANTENNA PERFORMANCE AT EACH STAGE OF MINIATURIZATION

\begin{tabular}{|l|l|l|l|}
\hline \multicolumn{1}{|c|}{ Stage } & $\begin{array}{c}\text { Frequency } \\
\text { (MHz) }\end{array}$ & $\begin{array}{c}\text { Gain } \\
\text { (dB) }\end{array}$ & ka \\
\hline Patch on ceramic & 4775 & -2.41 & 0.38 \\
\hline Straight slots & 3250 & -13.1 & 0.30 \\
\hline Vertical conductor sections & 2600 & -14.4 & 0.23 \\
\hline Bended slots and inductor & 2445 & -14.8 & 0.22 \\
\hline Tag on RPW & 2480 & -14.6 & 0.22 \\
\hline
\end{tabular}

\section{PERFORMANCE SIMULATIONS AND RESULTS}

Finally, the effect of the system-on-package (SoP) is addressed. The package, battery, BLE PCB and RPW body are included in the simulation, as shown in Fig. 6. The package is modeled as a cover with relative permittivity of 4.4 and loss tangent of 0.02 , while the RPW body is modeled as a $25 \times 15$ $\mathrm{x} 10 \mathrm{~mm}^{3}$ box with relative permittivity of 7 and loss tangent of 0.27 [9].

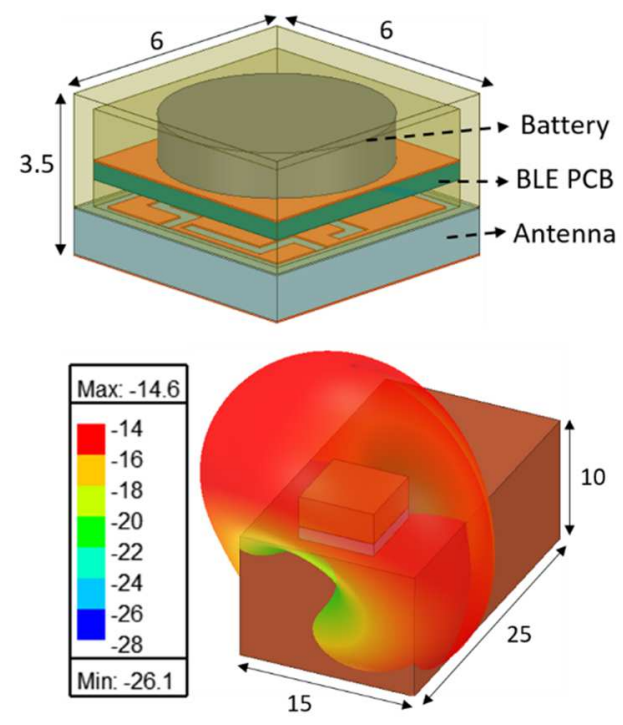

Fig. 6. System-on-package and simulation on modeled RPW. (Dimensions are in $\mathrm{mm}$ )

Fig. 7 displays the return loss and radiation pattern of the SoP on the RPW. The maximum gain is $-14.6 \mathrm{~dB}$. The frequency shifts up because of the dielectric loading effect of the package, while RPW body causes a gain attenuation of 1.7 $\mathrm{dB}$ at the bottom of the radiation pattern. As expected for a small antenna design, the radiation pattern exhibits a nearly omnidirectional shape. The antenna operates at $2.48 \mathrm{GHz}$ with a $10 \mathrm{~dB}$ bandwidth of $11 \mathrm{MHz}$, which is sufficient to cover the 39th advertisement channel at $2.48 \mathrm{GHz}$ as well as three data channels.
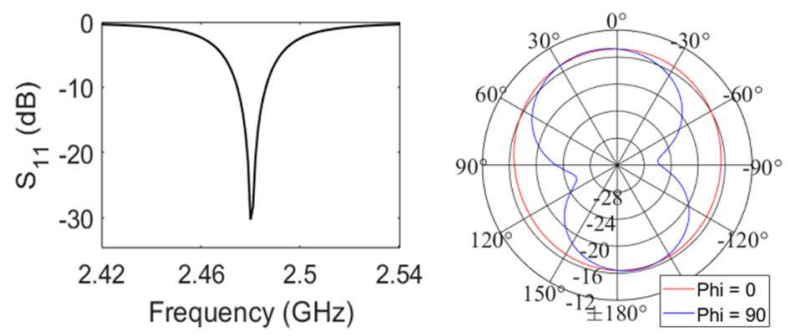

Fig. 7. Tag performance: return loss and radiation pattern.

Two ray ground reflection model [10] is used to estimate the detection range of the presented Bluetooth tag. On the receiver side, the chip sensitivity is $-96 \mathrm{dBm}$, and the typical gain of a Bluetooth antenna is $-2 \mathrm{~dB}$. On the transmitter side, the antenna gain is $-14.6 \mathrm{~dB}$ and the output power of the Bluetooth chip is $4 \mathrm{dBm}$. This translates to an estimated detection range of $78 \mathrm{~m}$.

\section{CONCLUSION}

An ultra-miniature Bluetooth enabled tracking tag for red palm weevil monitoring has been developed. Tag uses a modified patch antenna-on-package with a gain of $-14.6 \mathrm{db}$ and is detectable at a range of $78 \mathrm{~m}$ using Two-Ray ground reflection model. Size of the designed tag is smaller than similar tags previously reported in literature and detection range is suitable for red palm weevil monitoring.

\section{REFERENCES}

[1] Cant E.T, Smith A.D, Reynolds D.R and Osborne J.L., "Tracking butterfly flight paths across the landscape with harmonic radar," Proceedings of Royal Society B, vol. 272, no. 1565, pp. 785-790, 2005.

[2] H. Y. Al-Ayedh, " Evaluating a semi-synthetic diet for rearing the red palm weevil Rhynchophorus ferrugineus (Coleoptera: Curculionidae)," International Journal of Tropical Insect Science, vol. 31, no. 1-2, pp. 20-28, 2011.

[3] P. de Souza et al., "Low-Cost Electronic Tagging System for Bee Monitoring," Sensors, vol. 18, no. 7, p. 2124, 2018.

[4] N. Tahir and G. Brooker, "Toward the Development of Millimeter Wave Harmonic Sensors for Tracking Small Insects," IEEE Sensors Journal, vol. 15, no. 10, pp. 5669-5676, 2015.

[5] M. Kumari and S. M. R. Hasan, "A New CMOS Implementation for Miniaturized Active RFID Insect Tag and VHF Insect Tracking," IEEE Journal of Radio Frequency Identification, vol. 4, no. 2, pp. 124-136, 2020.

[6] H. Zhang and A. Shamim, "An Electrically Small Antenna in Package Design with Embedded Electronics for RPW Detection," 2020 IEEE International Symposium on Antennas and Propagation and North American Radio Science Meeting, pp. 33-34, 2020.

[7] M. Fallahpour and R. Zoughi, "Antenna Miniaturization Techniques: A Review of Topology- and Material-Based Methods," IEEE Antennas and Propagation Magazine, vol. 60, no. 1, pp. 38-50, 2018.

[8] John Volakis, Chi-Chih Chen and Kyohei Fujimoto, Small Antennas: Miniaturization Techniques \& Applications, 3rd ed., McGraw-Hill Education, pp. 132-134, 2010.

[9] Rita Massa et. al., "Wide Band Permittivity Measurements of Palm (Phoenix Canariensis) and Rhynchophorus ferrugineus (Coleoptera Curculionidae) for RF Pest Control," Journal of Microwave Power and Electromagnetic Energy, vol. 48, no. 3, pp. 158-169, 2014.

[10] T. S. Rappaport, Wireless communications: Principles and practice, 2nd ed., Prentice Hall, pp. 120, 2002. 